\title{
Radiation Effect on MHD Fully Developed Mixed Convection in a Vertical Channel with Asymmetric Heating
}

\author{
R. Patra ${ }^{1}$, S. Das ${ }^{2}$ and R. Nath Jana ${ }^{3 \dagger}$ \\ ${ }^{1,3}$ Department of Applied Mathematics, Vidyasagar University Midnapore 721 102, India \\ ${ }^{2}$ Department of Mathematics, University of Gour Banga, Malda 732 103, India
}

+Corresponding Author Email: jana261171@yahoo.co.in

(Received January 27, 2013; accepted April 3, 2013)

\begin{abstract}
Effects of radiative heat transfer on an MHD fully developed mixed convective flow of a viscous incompressible electrically conducting fluid through a vertical channel with asymmetric heating of walls in the presence of a uniform transverse magnetic field has been studied. An exact solution of the governing equations has been obtained in closed form. It is observed that the velocity field is greatly influenced by the radiative heat transfer as well as bouyancy forces. The induced magnetic field decreases near the cool wall and it increases near the hot wall of the channel with an increase in radiation parameter. Further, an increase in radiation parameter leads to a decrease in the fluid temperature in the channel. A limiting consideration of the solutions of the governing equations of the flow are analyzed for $R a \ll 1$.
\end{abstract}

Keywords: MHD mixed convective flow, Grashof number, radiation parameter, Prandtl number and asymmetric heating.

\section{NOMENCLATURE}

$\begin{array}{clll}B_{x} & \begin{array}{c}\text { induced magnetic field component along } x- \\ \text { direction }\end{array} & \operatorname{Pr} & \text { Prandtl number } \\ b_{x} & \begin{array}{c}\text { non-dimensional induced magnetic field } \\ \text { component }\end{array} & q_{r} & \text { radiative heat flux } \\ \vec{B} & \text { the magnetic field vector } & r_{T} & \text { temperature difference ratio } \\ e_{\lambda_{p}} & \text { planck function } & R a & \text { radiation parameter } \\ g & \text { acceleration due to gravity } & T & \text { fluid temperature } \\ G r & \text { Grashof number } & T_{0} & \text { temperature at entrance of channel } \\ G r_{0}, G r_{1} & \text { Critical Grashof numbers } & T_{1} & \text { temperature of hot wall } \\ K_{\lambda_{0}} & \text { absorption coefficient } & T_{2} & \text { temperature of cool wall } \\ k & \text { thermal conductivity } & u & \text { velocity component in } x \text {-direction } \\ M^{2} & \text { magnetic parameter } & u_{1} & \text { non-dimensional fluid velocity } \\ p & \text { fluid pressure } & (x, y) & \text { cartesian co-ordinates } \\ & & & \\ \alpha & \text { non-dimensional pressure gradient } & \theta & \text { dimensionless temperature } \\ \beta & \text { coefficient of thermal expansion } & \rho & \text { fluid density } \\ \mu_{e} & \text { magnetic permeability } & \rho_{0} & \text { fluid density at entrance of channel } \\ v & \text { kinematic viscosity } & \sigma & \text { conductivity of fluid } \\ \lambda & \text { wave length } & \tau_{0}, \tau_{1} & \text { shear stresses at cool and hot walls }\end{array}$

$\eta \quad$ non-dimensional width of the channel 


\section{INTRODUCTION}

Heat transfer in free and force convection in vertical channels occurs in any industrial processes and natural phenomena. Most of the interest in this subject is due to its applications, for instance, in the design of cooling systems or electronic devices, chemical processing equipment, microelectronic cooling and in the field of solar energy collections. Since some fluids can also emit and absorb thermal radiation, it is of interest to study the effects of magnetic field on the temperature distribution and heat transfer when the fluid is not only an electrical conductor but also when it is capable of emitting and absorbing radiation. Hence, heat transfer by thermal radiation is becoming of greater importance in space applications and higher operating temperatures. Ozisik(1989) has mentioned in an excellent review article that heat transfer by simultaneous radiation and convection has applications in numerous technological problems, including combustion, furnace design, the design of high-temperature gas-cooled nuclear reactors, nuclear-reactor safety, fluidized-bed heat exchagers, fire spreads, advanced enegy conservation devices such as open-cycle coal and natural-gas-fired MHD, solar ponds,solar collectors, natural convection in cavities and many others. On the other hand, it is worth mentionaing that heat transfer by simultaneous radiation and convection is very important in the context of space technology and processes involving high temperature. An excellent description of the fundamentals of thermal radiation has been presented in the book by Modest (2003). For a comprehensive treatment of the radiation transfer and the interractions with convection the interested reader can consult also the books by Sprrow and Cess 1970; Ozisik 1973 ; Siegel and Howell 1992). (Aung 1972; Aung et al.1972; Aung and Worku 1986; Barletta 2002 ; Boulama and Galanis 2004) deal with the evaluation of the temperature and velocity profiles for the vertical parallel-flow fully develoved regime. As is well known, heat exchangers technology involves convective flows in vertical channels. In most cases, these flows imply conditions of uniform heating of a channel, which can be modelled either by uniform wall temperature or uniform heat flux thermal boundary conditions. Many process in new engineering areas occur at high temperatures and knowledge of radiative heat transfer becomes very important for the design of the pertinent equipment. Nuclear power plants, gas turbines and various propulsion devices for aircraft, missiles satellites and space vehicles are examples of such engineering areas. Cogley et al.(1968) have studied the differential approximation for radiative heat transfer in a non-grey gas near equilibrium. The hydrodyamic fully developed laminar convective flow through a vertical channel in the optically thin limit has been studied by Greif et al. (1971) whereas Gupta and Gupta (1974) have studied the same problem in the presence of a transverse magnetic field. The effects of radiative heat transfer on MHD flows in vertical channel have been studied a number of researches. Datta and Jana
(1976) have discussed the effect of wall conductances on hydromagnetic convection of a radiating gas in a vertical channel. Ogulu and Motsa (2005) have studied the radiative heat transfer to magnetohydrodynamic Couette flow with variable wall temperature. Effect of radiation on unsteady free convection flow bounded by an oscillating plate with variable wall temperature have been described by Pathak et al.(2006). Sharma et al. (2007) have discussed the radiation effect on temperature distribution in three-dimensional Couette flow with suction / injection. The radiation effect on MHD free convection flow of a gas past a semi-infinite vertical plate have been studied by Takhar et al. (1996). The effects of wall conductance on MHD fully developed flow with asymmetric heating of the walls has been studied by Guria et al. (2007). Ghosh and Nandi (2000) have discussed magnetohydrodynamic fully developed combined convection flow between vertical plates heated asymmetrically. MHD fully developed mixed convection flow with asymmetric heating of the walls have been described by Ghosh et al. (2002). Pantokratoras (2006) has presented his results for a steady free convection flow between vertical parallel plates by considering different conditions on the wall temperature. The thermal radiation effect on fully developed mixed convection flow in a vertical channel have been examined by Grosan and Pop (2007). Suneetha et al. (2011) have presented the radiation and mass transfer effects on MHD free convective dissipative fluid in the presence of heat source/sink. Effects of thermal radiation on hydromagnetic flow due to a porous rotating disk with Hall effect have been studied by Anjali Devi and Uma Devi (2012). Baoku et al. (2012) have analyzed the influence of thermal radiation on a transient MHD Couette flow through a porous medium.

In the present paper, we study the effects of radiative heat transfer on MHD fully developed mixed convective flow in a vertical channel with the asymmetric heating of walls in the presence of a transverse magnetic field. We assume that the radiative heat flux follows a relation in an optically thin limit for a non-gray gas near equilibrium. The closed form solutions for velocity, temperature, shear stresses, rate of heat transfer and critical Grashof number are presented. Flow and heat transfer results for a range of values of the pertinent parameters have been reported. It is observed that the velocity field is greatly influenced by the radiative heat transfer as well as bouyancy forces. The induced magnetic field decreases near the cool wall and it increases near the hot wall of the vertical channel with an increase in radiation parameter. Further, an increase in radiation parameter leads to decrease the fluid temperature in the channel.

\section{FORMULATION OF THE PROBLEM AND ITS SOLUTION}

Consider a steady MHD fully developed mixed convective flow of a viscous incompressible electrically conducting fluid confined between 
vertical walls. The channel walls are at a distance $d$ apart. Choose a Cartesian co-ordinates system with $x$-axis in the upward direction along the cool wall in the direction of flow and the axis of $y$ is perpendicular to it. The wall at $y=0$ has a uniform temperature $T_{2}$ while the wall at $y=d$ is subjected to a uniform temperature $T_{1}$, where $T_{1}>T_{2}$. A uniform magnetic field of strength $B_{0}$ is imposed perpendicular to the channel walls. The flow is due to buoyancy force, difference in temperature and in the presence of pressure gradient. Since the channel walls are infinitely long along the $x$-direction, all physical quantities, are functions of $y$ only. The velocity components are $(u, v)$ relative to the Cartesian frame of reference.

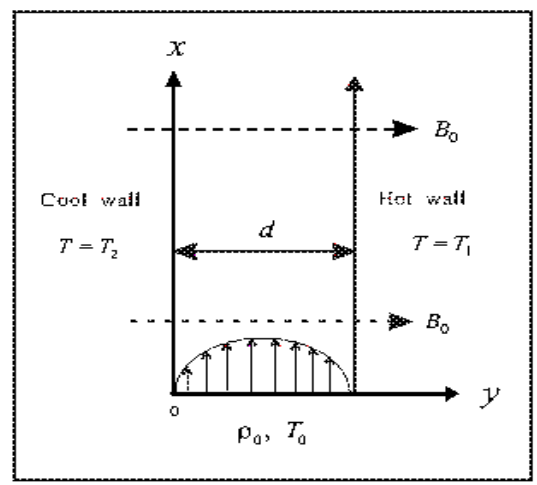

Fig. 1. Geometry of the problem

The Boussinesq approximation is assumed to hold and for the evaluation of the gravitational body force, the density is assumed to depend on the temperature according to the equation of state

$\rho=\rho_{0}\left[1-\beta\left(T-T_{0}\right)\right]$,

where $T$ is the fluid temperature, $\rho$ the fluid density, $\beta$ the coefficient of thermal expansion and $T_{0}$ and $\rho_{0}$ being the temperature and the density at the entrance of the channel.

The solenoidal equation $\nabla \cdot \vec{B}=0$ gives $B_{y}=$ constant $=B_{0}$ everywhere in the flow where $\vec{B} \equiv\left(B_{x}, B_{0}, 0\right)$. The flow being fully developed the relations $v=0, \frac{\partial v}{\partial y}=0$ and $\frac{\partial p}{\partial y}=0$ apply here, where $p$ is the fluid pressure. Therefore, the continuity equation gives $\frac{\partial u}{\partial x}=0$ and hence $u=u(y)$. Using the Boussinesq approximation (1), the momentum equation along $x$ - axis and the magnetic induction equation are

$$
\begin{aligned}
& v \frac{d^{2} u}{d y^{2}}+\mu_{e} B_{0} \frac{d B_{x}}{d y}+\rho_{0} g \beta\left(T-T_{0}\right)=\frac{d p}{d x}, \\
& \frac{d^{2} B_{x}}{d y^{2}}+\sigma \mu_{e} B_{0} \frac{d u}{d y}=0,
\end{aligned}
$$

and the energy equation is $0=k \frac{d^{2} T}{d y^{2}}-\frac{\partial q_{r}}{\partial y}$,

where $v$ is the kinematic viscosity, $\mu_{e}$ the magnetic permeability, $\sigma$ the conductivity of the fluid, $g$ the acceleration due to gravity, $k$ the thermal conductivity and $q_{r}$ the radiative heat flux.

It has been shown by Cogley et al.(1968) that in the optically thin limit for a non-gray gas near equilibrium, the following relation holds

$$
\frac{\partial q_{r}}{\partial y}=4\left(T-T_{0}\right) \int_{0}^{\infty} K_{\lambda_{0}}\left(\frac{\partial e_{\lambda h}}{\partial T}\right)_{0} d \lambda
$$

where $K_{\lambda_{0}}$ is the absorption coefficient, $\lambda$ is the wave length, $e_{\lambda p}$ is the Planck's function and subscript ' 0 ' indicates that all quantities have been evaluated at the temperature $T_{0}$ which is the temperature at entrance of the channel. On the use of (5), the Eq.(4) becomes

$0=k \frac{d^{2} T}{d y^{2}}-4 I\left(T-T_{0}\right)$,

where

$I=\int_{0}^{\infty} K_{\lambda_{0}}\left(\frac{\partial e_{\lambda h}}{\partial T}\right)_{0} d \lambda$

In asymmetric heating of the walls, the velocity, magnetic and temperature boundary conditions are respectively,

$u=0, T=T_{2}$ and $B_{x}=0$ at $y=0$,

$u=0, T=T_{1}$ and $B_{x}=0$ at $y=d$,

assuming the walls are electrically non-conducting.

Introducing the non-dimensional variables

$\eta=\frac{y}{d}, u_{1}=\frac{u d}{v}, b_{x}=\frac{B_{x}}{\sigma \mu_{e} v B_{0}}, \theta=\frac{T-T_{0}}{T_{1}-T_{0}}$,

Eqs. (2), (3) and (6) become

$\frac{d^{2} u_{1}}{d \eta^{2}}+M^{2} \frac{d b_{x}}{d \eta}+G r \theta+\alpha=0$,

$\frac{d^{2} b_{x}}{d \eta^{2}}+\frac{d u_{1}}{d \eta}=0$,

$\frac{d^{2} \theta}{d \eta^{2}}-R a \theta=0$,

where $\quad M=B_{0} \mu_{0} d\left(\frac{\sigma}{\rho_{0} v}\right)^{\frac{1}{2}}$ is the Hartmann number, $G r=\frac{g \beta\left(T_{2}-T_{1}\right) d^{3}}{v^{2}}$ the Grashof number and $R a=\frac{4 I d^{2}}{k}$ the radiation parameter and $\alpha=\frac{d^{3}}{\rho v^{2}}\left(-\frac{\partial p}{\partial x}\right)$ the non-dimensional pressure gradient.

The boundary conditions given by (7) become

$u_{1}=0, \theta=r_{T}$ and $b_{x}=0$ at $\eta=0$

$u_{1}=0, \theta=1$ and $b_{x}=0$ at $\eta=1$,

where $r_{T}=\frac{T_{2}-T_{0}}{T_{1}-T_{0}}$ is the temperature difference ratio.

Solution of Eqs. (9) to (11) subject to the boundary 
conditions (12) are

$$
\theta(\eta)= \begin{cases}\frac{\sinh \sqrt{R a} \eta}{\sinh \sqrt{R a}}+r_{T} \frac{\sinh \sqrt{R a}(1-\eta)}{\sinh \sqrt{R a}}, & R a \neq M^{2} \\ \frac{\sinh M \eta}{\sinh M}+r_{T} \frac{\sinh M(1-\eta)}{\sinh M}, & R a=M^{2},\end{cases}
$$$$
\int\left(\frac{\alpha}{M^{2}}+c\right)\left\{1-\frac{\sinh M \eta}{\sinh M}-\frac{\sinh M(1-\eta)}{\sinh M}\right\}
$$

$$
u_{1}(\eta)= \begin{cases}+\frac{G r}{R a-M^{2}}\left[\frac{\sinh M \eta}{\sinh M}+r_{T} \frac{\sinh M(1-\eta)}{\sinh M}\right\} & \\ \left.-\left\{\frac{\sinh \sqrt{R a} \eta}{\sinh \sqrt{R a}}+r_{T} \frac{\sinh \sqrt{R a}(1-\eta)}{\sinh \sqrt{R a}}\right\}\right], & R a \neq M^{2} \\ \left(\frac{\alpha}{M^{2}}+c\right)\left\{1-\frac{\sinh M \eta}{\sinh M}-\frac{\sinh M(1-\eta)}{\sinh M}\right\} & \\ -\frac{G r}{2 M \sinh M}\left[\left(r_{T}-\cosh M\right) \frac{\sinh M \eta}{\sinh M}\right. & R a=M^{2}, \\ \left.+\eta\left\{\cosh M \eta-r_{T} \cosh M(1-\eta)\right\}\right], & (14)\end{cases}
$$

$$
b_{x}(\eta)=\left\{\begin{array}{l}
c_{1}+c \eta-\left(\frac{\alpha}{M^{2}}+c\right)\left\{\eta-\frac{\cosh M \eta}{M \sinh M}+\frac{\cosh M(1-\eta)}{M \sinh M}\right\} \\
-\frac{G r}{R a-M^{2}}\left[\left\{\frac{\cosh M \eta}{M \sinh M}-r_{T} \frac{\cosh M(1-\eta)}{M \sinh M}\right\}\right. \\
\left.-\left\{\frac{\cosh \sqrt{R a} \eta}{\sqrt{R a} \sinh \sqrt{R a}}-r_{T} \frac{\cosh \sqrt{R a}(1-\eta)}{\sqrt{R a} \sinh \sqrt{R a}}\right\}\right], R a \neq M^{2} \\
c_{1}+c \eta-\left(\frac{\alpha}{M^{2}}+c\right)\left\{\eta-\frac{\cosh M \eta}{M \sinh M}+\frac{\cosh M(1-\eta)}{M \sinh M}\right\} \\
+\frac{G r}{2 M \sinh M}\left[\left(r_{T}-\cosh M\right) \frac{\cosh M \eta}{M \sinh M}\right. \\
+\frac{\eta}{M}\left\{\sinh M \eta+r_{T} \sinh M(1-\eta)\right\} \\
\left.-\frac{1}{M^{2}}\left\{\cosh M \eta-r_{T} \cosh M(1-\eta)\right\}\right], R a=M^{2}
\end{array}\right.
$$

where

$$
c_{1}= \begin{cases}\frac{\alpha}{2 M^{2}}+\frac{G r\left(1-r_{T}\right)}{2\left(R a-M^{2}\right)}\left[\frac{1+\cosh M}{M \sinh M}-\frac{1+\cosh \sqrt{R a}}{\sqrt{R a} \sinh \sqrt{R a}}\right], & R a \neq M^{2} \\ \frac{\alpha}{2 M^{2}}+\frac{G r\left(1-r_{T}\right)}{4 M^{3} \sinh ^{2} M}(1+\cosh M)(M+\sinh M), & R a=M^{2},\end{cases}
$$

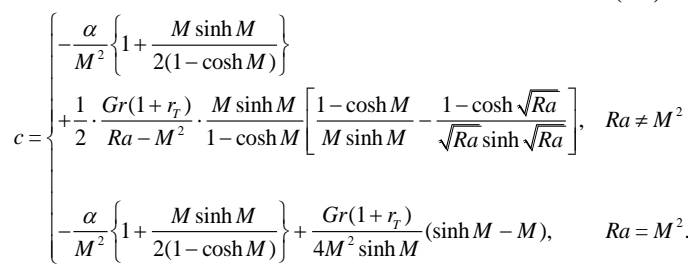

and $\alpha$ is given by (19).

It is seen from the expressions (13)-(15) that the velocity field and induced magnetic field depend on the Grashof number $G r$, whereas the temperature distribution is independent of $G r$. In the absence of radiation ( $R a=0$ ), the velocity distribution and the induced magnetic field coincide with Guria et al.(2007) in the case of without wall conductance.

\section{EXPRESSION FOR PRESSURE GRADIENT}

It is noticed from the Eqs. (14) and (15) that the parameter $\alpha$ is still to be evaluated. Using the rate of mass flow

$\int_{0}^{1} u_{1} d \eta=1$,

we have, on the substitution of the value of $u_{1}$, which is obtained from Eq. (14) as

$$
\alpha= \begin{cases}\frac{2 M^{2}(\cosh M-1)}{M \sinh M+2(1-\cosh M)} & \\ +\frac{G r\left(1+r_{T}\right)}{R a-M^{2}} \cdot \frac{M^{3} \sinh M}{M \sinh M+2(1-\cosh M)} & R a \neq M^{2} \\ \times\left[\frac{1-\cosh M}{M \sinh M}-\frac{1-\cosh \sqrt{R a}}{\sqrt{R a} \sinh \sqrt{R a}}\right], & \\ \frac{2 M^{2}(\cosh M-1)}{M \sinh M+2(1-\cosh M)} & R a=M^{2} . \\ \times\left[1-\frac{1}{4} \operatorname{Gr}\left(1+r_{T}\right) \frac{\sinh M-M}{M^{2} \sinh M}\right], & \end{cases}
$$

\section{RESULTS AND DISCUSSION}

To study the effects of radiative heat transfer and the magnetic field on the MHD fully developed flow with asymmetric heating of the walls, the dimensionless velocity $u_{1}$, the induced magnetic filed $b_{x}$ and temperature distribution $\theta$ are depicted graphically against $\eta$ for several values of radiation parameter $R a$, magnetic parameter $M^{2}$, bounyancy parameter $G r$ and temperature difference ratio $r_{T}$ in Figs.2 to 13. Figures. 2 and 3 depicts the effects of the radiative heat transfer and magnetic field on the velocity field. It is seen from Fig. 2 that the fluid velocity $u_{1}$ increases in the range $0 \leq \eta \leq 0.38$ while it decreases in the range $0.38<\eta \leq 0.86$ and again it increases in the range $0.86<\eta \leq 1$ with an increase in radiation parameter $R a$. It is manifested that there is a closeness of the curves near the hot wall. Fig.3 reveals that the fluid velocity $u_{1}$ increases in the range $0 \leq \eta \leq 0.42$ while it decreases in the range $0.42<\eta \leq 1$ with an increase in magnetic parameter $M^{2}$. It means that the Lorentz force imposed by a transverse magnetic field to an electrically conducting fluid, which slows down the fluid motion near the hot wall and enhances the fluid motion near cool wall. It is also manifested that there is a closeness of the curves near the hot wall. It is noticed from Fig. 4 that the fluid velocity $u_{1}$ decreases in the region $0 \leq \eta \leq 0.53$ and it increases in the region $0.53<\eta \leq 1$ with increase in Grashof number $G r$. Fig.5 shows that with an increase in $r_{T}$, the fluid velocity $u_{1}$ increases in the region $0 \leq \eta \leq 0.53$ and it decreases in the region $0.53<\eta \leq 1$. It is 
R. Patra et al. / JAFM, Vol. 7, No. 3, pp. 503-512, 2014.

observed from Fig.6 that the induced magnetic field $b_{x}$ decreases at any point near the cool wall and it increases near the hot wall with an increase in $R a$. Fig.7 shows that the induced magnetic field $b_{x}$ decreases with an increase in $M^{2}$. It is seen from Fig. 8 that the induced magnetic field $b_{x}$ increases with an increase in $G r$. Fig.9 reveals that the induced magnetic field $b_{x}$ decreases with an increase in $r_{T}$. The temperature profiles have been drawn for different values of $R a$ and $r_{T}$ in Figs.10 and 11. It is seen from Figs. 10 and 11 that the fluid temperature $\theta$ decreases with an increase in radiation parameter $R a$ while it increases with an increase in temperature difference ratio parameter $r_{T}$.

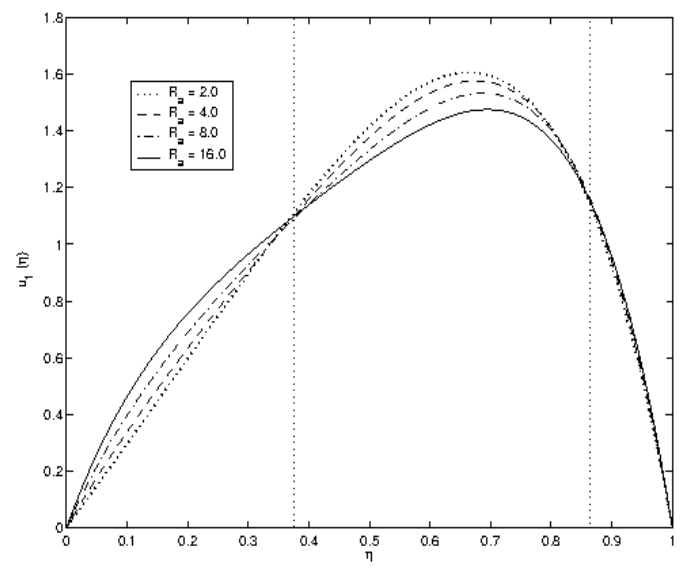

Fig. 2. Variation of velocity for different $R a$ when $M^{2}=5, r_{T}=0.4$ and $G r=100$

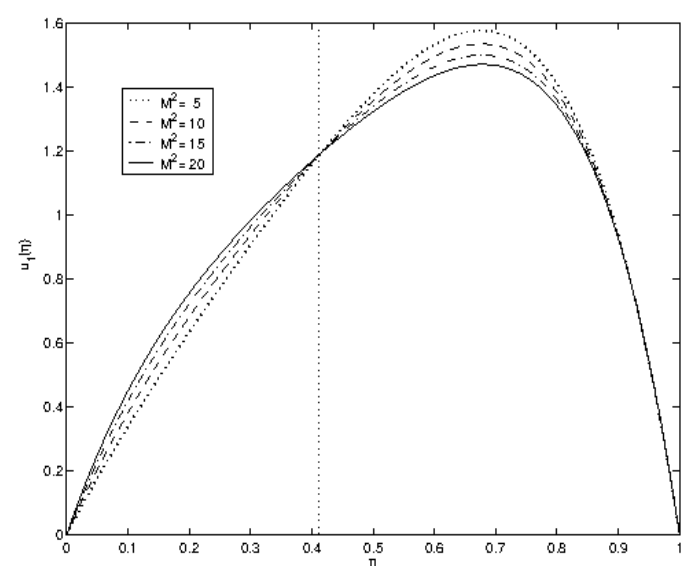

Fig. 3. Variation of velocity for different $M^{2}$ when $R a=4, r_{T}=0.4$ and $G r=100$ 


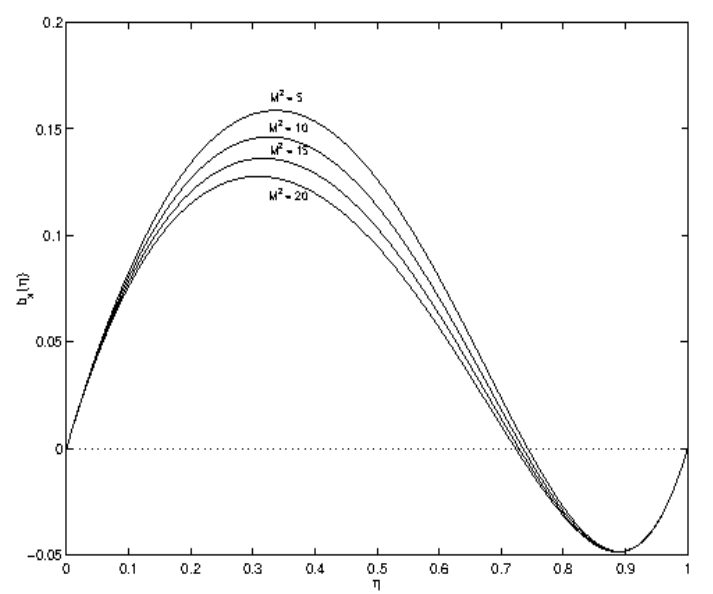

Fig. 7. Variation of induced magnetic field for different $M^{2}$ when $R a=4, r_{T}=0.4$ and $G r=100$

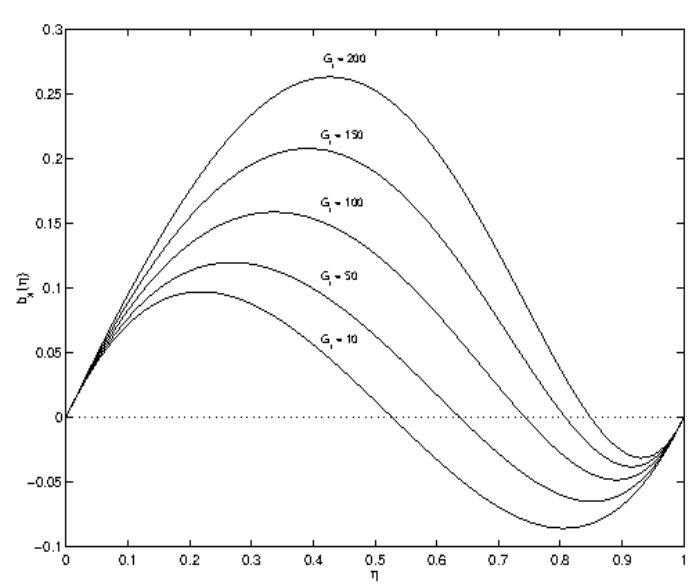

Fig. 8. Variation of induced magnetic field for different $G r$ when $M^{2}=5, R a=4$ and $G r=100$

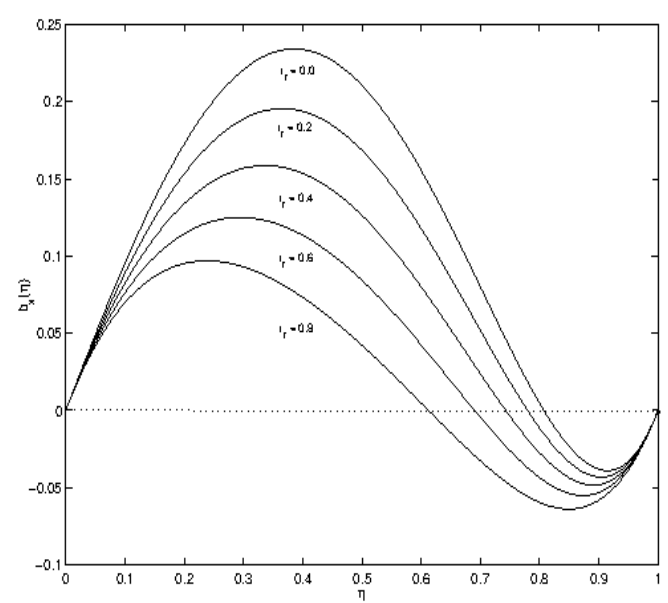

Fig. 9. Variation of induced magnetic field for different $r_{T}$ when $M^{2}=5, R a=4$ and $G r=100$

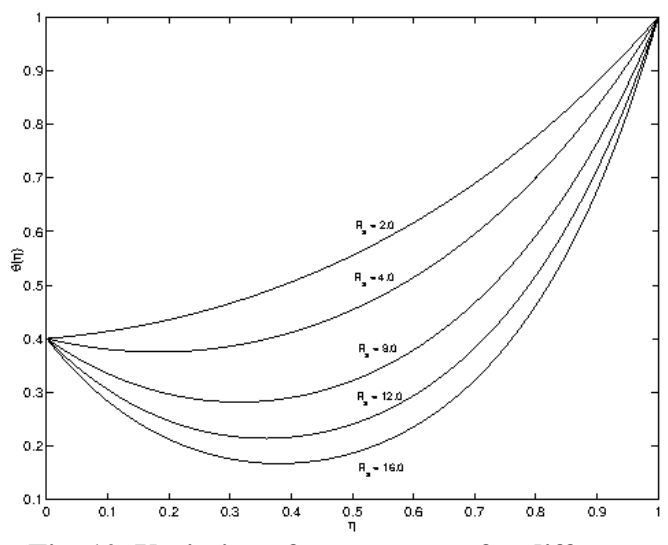

Fig. 10. Variation of temperature for different $R a$ when $r_{T}=0.4$

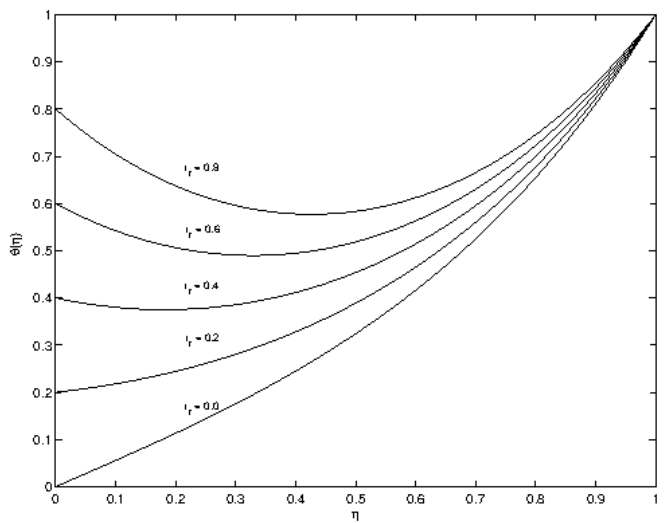

Fig. 11. Variation of temperature for different $r_{T}$ when $R a=4$

One of the important characteristics of this problem is the shear stress at the walls. The non-dimensional shear stress at the cool wall $(\eta=0)$ and hot wall $(\eta=1)$ are respectively given by

$$
\tau_{x_{0}}=\left(\frac{d u_{1}}{d \eta}\right)_{\eta=0} \text { and } \tau_{x_{1}}=\left(\frac{d u_{1}}{d \eta}\right)_{\eta=1} \text {, }
$$

where

$$
\left(\frac{d u_{1}}{d \eta}\right)_{\eta=0}=\left\{\begin{array}{l}
\frac{M^{2}(\cosh M-1)}{M \sinh M+2(1-\cosh M)} \\
\times\left[1+\frac{G r\left(1+r_{T}\right)}{R a-M^{2}}\left(\frac{1-\cosh M}{M \sinh M}-\frac{1-\cosh \sqrt{R a}}{\sqrt{R a} \sinh \sqrt{R a}}\right)\right] \\
+\frac{G r}{R a-M^{2}} \\
\times\left[\frac{M\left(1-r_{T} \cosh M\right)}{\sinh M}-\frac{\sqrt{R a}\left(1-r_{T} \cosh \sqrt{R a}\right)}{\sinh \sqrt{R a}}\right], R a \neq M^{2} \\
\frac{M^{2}(\cosh M-1)}{M \sinh M+2(1-\cosh M)} \\
\times\left[1+G r\left(1+r_{T}\right) \frac{(1-\cosh M)(\sinh M-M)}{2 M^{3} \sinh ^{2} M}\right] \\
-\frac{G r}{2 M \sinh M} \\
\times\left[\left(r_{T}-\cosh M\right) \frac{M}{\sinh M}+\left(1-r_{T} \cosh M\right)\right], R a=M^{2}
\end{array}\right.
$$

and 


$$
\left(\frac{d u_{1}}{d \eta}\right)_{\eta=1}=\left\{\begin{array}{l}
\frac{M^{2}(1-\cosh M)}{M \sinh M+2(1-\cosh M)} \\
\times\left[1+\frac{G r\left(1+r_{T}\right)}{R a-M^{2}}\left(\frac{1-\cosh M}{M \sinh M}-\frac{1-\cosh \sqrt{R a}}{\sqrt{R a} \sinh \sqrt{R a}}\right)\right] \\
+\frac{G r}{R a-M^{2}} \\
\times\left[\frac{M\left(\cosh M-r_{T}\right)}{\sinh M}-\frac{\sqrt{R a}\left(\cosh \sqrt{R a}-r_{T}\right)}{\sinh \sqrt{R a}}\right], R a \neq M^{2} \\
\frac{M^{2}(1-\cosh M)}{M \sinh M+2(1-\cosh M)} \\
\times\left[1+G r\left(1+r_{T}\right) \frac{(1-\cosh M)(\sinh M-M)}{2 M^{3} \sinh ^{2} M}\right] \\
-\frac{G r}{2 M \sinh M} \\
\times\left[\left(r_{T} \cosh M-1\right) \frac{M}{\sinh M}+\left(\cosh M-r_{T}\right)\right], R a=M^{2}
\end{array}\right.
$$

The values of non-dimensional shear stresses at the walls $\eta=0$ and $\eta=1$ are shown graphically against $r_{T}$ for different values of $R a$ and $M^{2}$ in Figs.12 and 13. It is observed from Fig.12 that for fixed values of $M^{2}$ and $G r$ the shear stress $\tau_{x_{0}}$ at the cool wall increases while the shear stress $\tau_{x_{1}}$ at the hot wall decreases with an increase in radiation parameter $R a$. On the other hand, Fig.13 shows that the shear stress $\tau_{x_{0}}$ at the cool wall increases while the shear stress $\tau_{x_{1}}$ at the right wall decreases with increase in $M^{2}$. It is interesting to note that the shear stress due to the flow does not vanish at the walls $\eta=0$ and $\eta=1$ when buoyancy forces $G r=0$. Thus we arrive an interesting conclusion that there is no flow reversal in the absence of the buoyancy forces.

The shear stresses at the cool and hot wall vanish if

$$
G r_{0}=\left\{\begin{array}{cl}
\frac{\left(R a-M^{2}\right) M^{2}(1-\cosh M)}{(A+B)[M \sinh M+2(1-\cosh M)]}, & R a \neq M^{2} \\
\frac{2 M^{2}(1-\cosh M)}{(A+B)[M \sinh M+2(1-\cosh M)]}, & R a=M^{2},
\end{array}\right.
$$$$
G r_{1}=\left\{\begin{array}{cc}
\frac{\left(R a-M^{2}\right) M^{2}(1-\cosh M)}{\left(A_{1}+B_{1}\right)[M \sinh M+2(1-\cosh M)]}, & R a \neq M^{2} \\
\frac{2 M^{2}(1-\cosh M)}{\left(A_{1}+B_{1}\right)[M \sinh M+2(1-\cosh M)]}, & R a=M^{2},
\end{array}\right.
$$

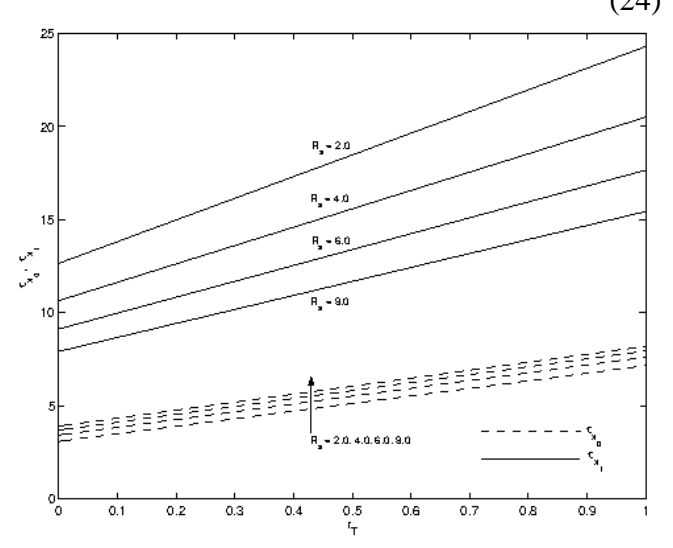

Fig. 12. Shear stress for different $R a$ when $M^{2}=5, R a=4$ and $G r=50$

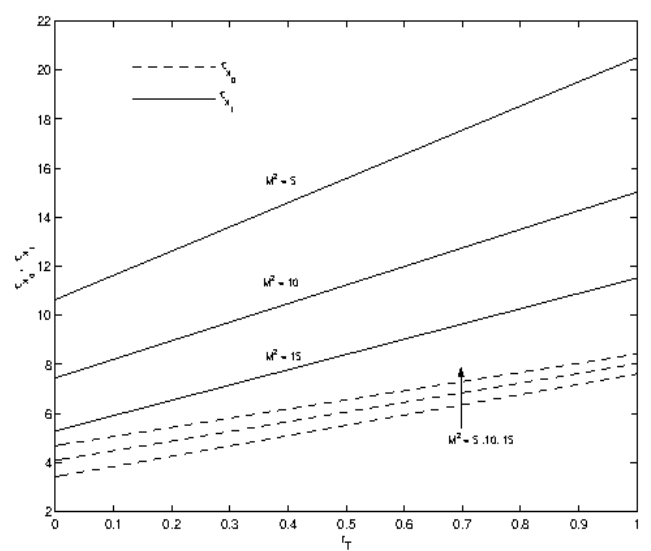

Fig. 13. Shear stress for different $M^{2}$ when $M^{2}=5, r_{T}=0.4$ and $G r=50$

Table 1 The values of Critical Grashof number $G r_{0} \times 10^{-3}$ at the cool wall. $G r_{0}$

\begin{tabular}{|c|c|c|c|c|c|c|}
\hline & \multicolumn{3}{|c|}{$R a$} & \multicolumn{3}{c|}{$r_{T}$} \\
\hline$M^{2}$ & 2 & 4 & 6 & 0 & 0.2 & 0.4 \\
& & & & & & \\
\hline 5 & 0.1821947 & 0.2327067 & 0.2946396 & 0.1056287 & 0.1453024 & 0.2327067 \\
10 & 0.2102455 & 0.2710896 & 0.347127 & 0.1214226 & 0.1677217 & 0.2710896 \\
15 & 0.2385835 & 0.3102064 & 0.4012581 & 0.1373196 & 0.1903684 & 0.3102064 \\
20 & 0.2671151 & 0.3498836 & 0.4567396 & 0.1532741 & 0.2131661 & 0.3498836 \\
\hline
\end{tabular}


R. Patra et al. / JAFM, Vol. 7, No. 3, pp. 503-512, 2014.

Table 2 The values of Critical Grashof number $G r_{1} \times 10^{-3}$ at the hot wall.

\begin{tabular}{|c|c|c|c|c|c|c|}
\hline & \multicolumn{3}{|c|}{$R a$} & \multicolumn{3}{c|}{$r_{T}$} \\
\hline$M^{2}$ & 2 & 4 & 6 & 0 & 0.2 & 0.4 \\
\hline 5 & 0.1197925 & 0.109435 & 0.1033845 & 0.07737131 & 0.09065144 & 0.109435 \\
10 & 0.1361429 & 0.123871 & 0.1167035 & 0.08797246 & 0.10288010 & 0.123871 \\
15 & 0.1524667 & 0.138243 & 0.1299393 & 0.09855560 & 0.11507350 & 0.138243 \\
20 & 0.1687417 & 0.152538 & 0.1430853 & 0.10910590 & 0.1272174 & 0.1525389 \\
\hline
\end{tabular}

The values of the critical Grashof numbers $G r_{0}$ and $G r_{1}$ due to the flow at the cool wall $\eta=0$ and hot wall $\eta=1$ are entered in the Tables 1 and 2 for different values of magnetic parameter $M^{2}$, radiation parameter $R a$ and the temperature difference ratio parameter $r_{T}$. It is seen from the Table 1 that the critical Grashof number $G r_{0}$ at the cool wall $(\eta=0)$ due to the flow increases with increase in either $R a$ or $r_{T}$ or $M^{2}$. Table 2 shows that the the critical Grashof number $G r_{1}$ at the hot wall $(\eta=1)$ due to the flow increases with an increase in either $M^{2}$ or $r_{T}$. On the other hand, with increase in the radiation parameter $R a$, the critical Grashof number $G r_{1}$ decreases.

In the absence of radiative heat transfer $(R a=0)$, the critical Grashof numbers $G r_{0}$ and $G r_{1}$ are given by

$$
G r_{0}=\frac{2 M^{4}(\cosh M-1)}{\left(1-r_{T}\right)\left[M^{2}(1+\cosh M)+4 \cosh M-4(1+\cosh M)\right]},
$$

$G r_{1}=-G r_{0}$,

which are identical with the Eq. (39) of Guria et al. (2007).

\subsection{Limiting Case}

Now, we discuss the case when the radiation parameter is $R a \ll 1$. In this case, Eqs. (13) to (15) become

$$
\begin{aligned}
& \left.\theta(\eta)=\eta+\frac{R a}{6}\left(\eta^{3}-\eta\right)+r_{T}[1-\eta)+\frac{R a}{6} \eta(1-\eta)(\eta-2)\right], \\
& u_{1}(\eta)=\left(\frac{\alpha}{M^{2}}+c\right)\left\{1-\frac{\sinh M \eta}{\sinh M}-\frac{\sinh M(1-\eta)}{\sinh M}\right\} \\
& +\frac{G r}{R a-M^{2}}\left[\left\{\frac{\sinh M \eta}{\sinh M}+r_{T} \frac{\sinh M(1-\eta)}{\sinh M}\right\}\right. \\
& \left.\left.-\left[\eta+\frac{R a}{6}\left(\eta^{3}-\eta\right)+r_{T}\{1-\eta)+\frac{R a}{6} \eta(1-\eta)(\eta-2)\right\}\right]\right] \\
& b_{x}(\eta)=\frac{\alpha}{2 M^{2}}+\frac{G r\left(1-r_{T}\right)}{2\left(R a-M^{2}\right)} \cdot \frac{1+\cosh M}{M \sinh M}+c \eta \\
& -\left(\frac{\alpha}{M^{2}}+c\right)\left\{\eta-\frac{\cosh M \eta}{M \sinh M}+\frac{\cosh M(1-\eta)}{M \sinh M}\right\} \\
& -\frac{G r}{R a-M^{2}}\left\{\frac{\cosh M \eta}{M \sinh M}-r_{T} \frac{\cosh M(1-\eta)}{M \sinh M}\right\} \\
& -\frac{3 G r}{\left(R a-M^{2}\right)(R a+6)}\left\{\frac{1}{2}\left(1-r_{T}\right)-\eta^{2}+r_{T}\left(1-2 \eta+\eta^{2}\right)\right\}(29)
\end{aligned}
$$

where

$c=\left[-\frac{\alpha}{M^{2}}+\frac{G r\left(1+r_{T}\right)}{2\left(R a-M^{2}\right)}\right]\left[\frac{M \sinh M+2(1-\cosh M)}{2(\cosh M-1)}\right]$, $\alpha=\frac{2 M^{2}(\cosh M-1)}{M \sinh M+2(1-\cosh M)}+\frac{M^{2} G r\left(1+r_{T}\right)}{2\left(\operatorname{Ra}-M^{2}\right)}(30)$

In limit $R a \rightarrow 0$, Eqs. (27) to (28) for the temperature, the velocity and the induced magnetic field yield respectively

$\theta(\eta)=\eta+r_{T}(1-\eta)$,

$u_{1}(\eta)=\frac{1}{M^{2}}\left[\left(G r r_{T}+\alpha+M^{2} c\right)\left\{1-\frac{\sinh M \eta}{\sinh M}-\frac{\sinh M(1-\eta)}{\sinh M}\right\}\right.$

$\left.+G r\left(1-r_{T}\right)\left(\eta-\frac{\sinh M \eta}{\sinh M}\right)\right]$,

$b_{x}(\eta)=-\frac{1}{M^{2}}\left[\left(G r r_{T}+\alpha+M^{2} c\right)\left\{\eta-\frac{\cosh M \eta}{M \sinh M}+\frac{\cosh M(1-\eta)}{M \sinh M}\right\}\right.$

$\left.+G r\left(1-r_{T}\right)\left(\frac{1}{2} \eta^{2}-\frac{\cosh M \eta}{M \sinh M}\right)\right]+c \eta+c_{1}$,

where

$c=\left[r_{T} G r+\alpha+\frac{1}{2} G r\left(1-r_{T}\right)\right] \frac{[M \sinh M+2(1-\cosh M)]}{2 M^{2}(\cosh M-1)}$

$c_{1}=\frac{1}{2 M^{2}}\left[\left(r_{T} G r+\alpha\right)+\frac{1}{2} \operatorname{Gr}\left(1-r_{T}\right)\left\{\frac{M \sinh M-2(1+\cosh M)}{M \sinh M}\right\}\right]$

$\alpha=\frac{2 M^{2}(\cosh M-1)}{M \sinh M+2(1-\cosh M)}-\frac{1}{2} G r\left(1+r_{T}\right)$.

The velocity and the induced magnetic field given by (32) and (33) coincide with Eqs. (14) and (15) of Guria et al. (2007) in the case of without wall conductance.

\section{CONCLUSION}

An MHD fully developed mixed convection in a vertical channel in the presence of radiation have been studied. It is seen that the fluid velocity is strongly affected by the radiative heat transfer as well as bouyancy force. The induced magnetic field decreases near the cool wall and it increases near the hot wall of the channel with an increase in radiation parameter. Radiation decreases the fluid temperature. It is interesting to note that the shear stress due to the flow does not vanish at the channel walls when $G r=0$ and hence there is no flow reversal in the absence of the buoyancy force. This model finds applications in the design of cooling systems, chemical processing equipments and the field of solar energy collections.

\section{REFERENCES}

Anjali Devi, S.P. and R. Uma Devi (2012). Effects of thermal radiation on hydromagnetic flow 
due to a porous rotating disk with Hall effect. J. Applied Fluid Mechanics 5(2), 1-7.

Aung, W. and G. Worku (1986). Developing flow and flow reversal in a vertical channel with asymmetric wall temperature. J.Heat Transfer 108, 299-304.

Aung, W. and G.Worku (1986). Theory of fully develoed, combined convection including flow reversal. J.Heat Transfer 108, 485-488.

Aung, W., L.S. Fletcher and V. Sernas (1972). Developing laminer free convection between vertical flat plates asymmetric heating. Int.J.Heat Mass Transfer 15, 2293-2308.

Aung, W. (1972). Fully developed laminer free convection between vertical plates heated asymmetrically. Int.J.Heat Mass Transfer 15, $1577-1580$

Baoku, I. G., C. Israel-Cookey and B. I. Olajuwon (2012). Influence of thermal radiation on a transient MHD Couette flow through a porous medium. J. Applied Fluid Mechanics 5(1), 8187.

Barletta, A. (2002). Fully developed mixed convection and flow reversal in a vertical rectangular duct with unifrom wall heat flux. Int.J. Heat Mass Transfer 45, 641-654.

Boulama, K. and N. Galanis (2002). Fully developed mixed convection and flow reversal in a vertical rectangular duct with uniform wall heat flux. Int.J. Heat Mass Transfer 45, 641654.

Cogley, A.C., W.C.Vincenti and S.E. Gilles (1968). Differential approximation for radiative transfer in a non-grey gas near equilibrium. AIAAJ ,16, 551-553.

Datta, N. and R.N. Jana (1976). Effects of wall conductances on hydromagnetic convection of a radiating gas in a vertical channel. Int. J. Heat Mass Transfer, 9, 1015-1019.

Ghosh, S.K, I. Pop and D.K. Nandi (2002). MHD fully developed mixed convection flow with asymmetric heating of the walls. Int. J. Appl. Mech. and Engineering 7, 1211.

Ghosh, S.K. and D.K. Nandi (2000). Magnetohydrodynamic fully developed combined convection flow between vertical plates heated asymmetrically. Journal Technical Physics ,41, 173.

Greif, R., I.S. Habib and J.C. Lin (1971). Laminar convection of a radiating gas in a vertical channel. J. Fluid Mech., 46(3), 513-520.

Grosan, T. and I. Pop (2007). Thermal radiation effect on fully develoved mixed convection flow in a vertical channel Technische
Mechanik 27(1), 37-47.

Gupta, P.S. and A. S. Gupta (1974). Radiation effect on hydromagnetic convection in a vertical channel. Int.J. Heat Mass Transfer 17, $1437-1442$.

Guria, M., Das, B.K., Jana, R.N. and Ghosh, S.K. (2007). Effects of wall conductance on MHD fully developed flow with asymmetric heating of the walls. Int.J.Fluid Mech.Research 34(6), 521-534.

Modest, M. F. (2003). Radiative Heat Transfer ( $2^{\text {nd }}$ edition). Academic Press, New York.

Ogulu, A. and S. Motsa (2005). Radiative heat transfer to magnetohydrodynamic Couette flow with variable wall temperature Physica Scripta 71, 336-339.

Ozisik, M.N. (1973). Radiative Transfer and Interactions with Conduction and Convection. Wiley, New York.

Ozisik, M.N. (1987). Interaction of radiation with convection. In: Handbook of Single-Phase Convection Heat Transfer (Kakac, S., Shah, R.K. and Aung W., eds.) . Wiley, New York.

Pantokratoras, A. (2006). Fully developed laminar free covection with variable thermophysical properties between two open-ended vertical parallel plates heated asymmetrically with large temperature differences. ASME J. Heat Tranfer (128), 405-408.

Pathak, G., C. H. Maheshwari and S. P. Gupta (2006). Effect of radiation on unsteady free convection flow bounded by an oscillating plate with variable wall temperature Int. J. Appl. Mech.and Engineering 2, 371-382.

Sharma, B.K., M. Agarwal and R.C. Chaudhary (2007). Radiation effect on temperature distribution in three-dimensional Couette flow with suction or injection. Appl. Math. Mech. (English Edition) 28(3), 309-316.

Siegel, R. and J.R. Howell (1992). Thermal Radiation Heat Transfer $\left(3^{\text {rd }}\right.$ edition). Hemisphere, New York.

Sparrow, E.M. and R. D. Cess (1970). Radiation Heat Transfer. Brooks/Cole, Belmont, California.

Suneetha, S., N. Bhaskar Reddy and V. Ramachandra Prasad (2011). Radiation and mass transfer effects on MHD free convective dissipative fluid in the presence of heat source/sink. J. Applied Fluid Mechanics 4(1), 107-113.

Takhar, H.S., S. R. Gorla and V.M.Soundalgekar (1996). Radiation effect on MHD free convection flow of a gas past a semi-infinite 
R. Patra et al. / JAFM, Vol. 7, No. 3, pp. 503-512, 2014.

vertical plate. Int. J. Num. Methods for Heat and Fluid Flow 2(6), 77.

\section{APPENDIX}

$A= \begin{cases}\frac{M^{2}\left(1+r_{T}\right)(\cosh M-1)}{M \sinh M+2(1-\cosh M)}\left[\frac{1-\cosh M}{M \sinh M}-\frac{1-\cosh \sqrt{R a}}{\sqrt{R a} \sinh \sqrt{R a}}\right], & R a \neq M^{2} \\ \frac{\left(1+r_{T}\right)(1-\cosh M)^{2}(M-\sinh M)}{M \sinh ^{2} M[M \sinh M+2(1-\cosh M)]} & R a=M^{2},\end{cases}$

$A_{1}= \begin{cases}\frac{M^{2}\left(1+r_{T}\right)(1-\cosh M)}{M \sinh M+2(1-\cosh M)}\left[\frac{1-\cosh M}{M \sinh M}-\frac{1-\cosh \sqrt{R a}}{\sqrt{R a} \sinh \sqrt{R a}}\right], & R a \neq M^{2} \\ \frac{\left(1+r_{T}\right)(1-\cosh M)^{2}(\sinh M-M)}{M \sinh ^{2} M[M \sinh M+2(1-\cosh M)]}, & R a=M^{2},\end{cases}$

$A_{1}= \begin{cases}\frac{M^{2}\left(1+r_{T}\right)(1-\cosh M)}{M \sinh M+2(1-\cosh M)}\left[\frac{1-\cosh M}{M \sinh M}-\frac{1-\cosh \sqrt{R a}}{\sqrt{R a} \sinh \sqrt{R a}}\right], & R a \neq M^{2} \\ \frac{\left(1+r_{T}\right)(1-\cosh M)^{2}(\sinh M-M)}{M \sinh ^{2} M[M \sinh M+2(1-\cosh M)]}, & R a=M^{2},\end{cases}$

$B_{1}= \begin{cases}\frac{M\left(\cosh M-r_{T}\right)}{\sinh M}-\frac{\sqrt{R a}\left(\cosh \sqrt{R a}-r_{T}\right)}{\sinh \sqrt{R a}}, & R a \neq M^{2} \\ \frac{M\left(1-r_{T} \cosh M\right)+\sinh M\left(r_{T}-\cosh M\right)}{M_{\sinh ^{2} M}}, & R a=M^{2},\end{cases}$

$B= \begin{cases}\frac{M\left(1-r_{T} \cosh M\right)}{\sinh M}-\frac{\sqrt{R a}\left(1-r_{T} \cosh \sqrt{R a}\right)}{\sinh \sqrt{R a}}, & R a \neq M^{2} \\ \frac{M\left(\cosh M-r_{T}\right)+\sinh M\left(r_{T} \cosh M-1\right)}{M \sinh ^{2} M}, & R a=M^{2},\end{cases}$ 